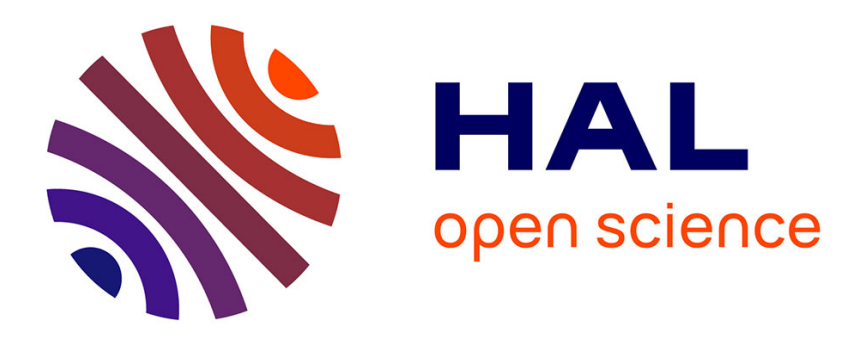

\title{
A stochastic reaction scheme for drug/metabolite interaction
}

F. Di Patti, D. Fanelli

\section{To cite this version:}

F. Di Patti, D. Fanelli. A stochastic reaction scheme for drug/metabolite interaction. Journal of Theoretical Biology, 2009, 259 (2), pp.382. 10.1016/j.jtbi.2009.03.031 . hal-00554598

\section{HAL Id: hal-00554598 \\ https://hal.science/hal-00554598}

Submitted on 11 Jan 2011

HAL is a multi-disciplinary open access archive for the deposit and dissemination of scientific research documents, whether they are published or not. The documents may come from teaching and research institutions in France or abroad, or from public or private research centers.
L'archive ouverte pluridisciplinaire HAL, est destinée au dépôt et à la diffusion de documents scientifiques de niveau recherche, publiés ou non, émanant des établissements d'enseignement et de recherche français ou étrangers, des laboratoires publics ou privés. 


\section{Author's Accepted Manuscript}

A stochastic reaction scheme for drug/metabolite interaction

F. Di Patti, D. Fanelli

PII:

S0022-5193(09)00139-8

DOI: doi:10.1016/j.jtbi.2009.03.031

Reference: YJTBI 5515

To appear in: Journal of Theoretical Biology

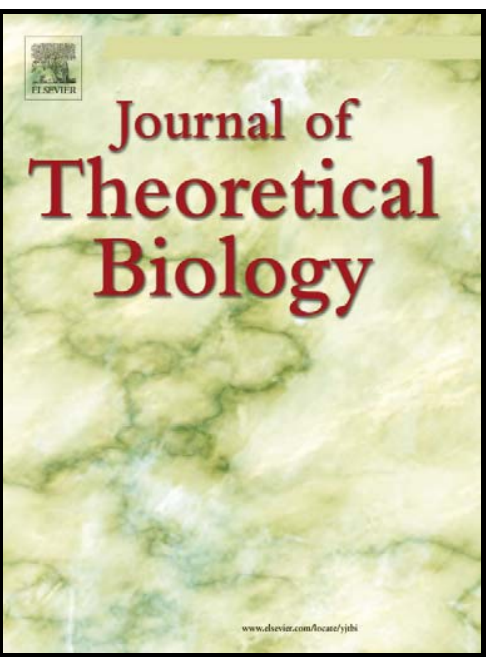

www.elsevier.com/locate/yjtb

Received date: 8 January 2009

Accepted date: $\quad 24$ March 2009

Cite this article as: F. Di Patti and D. Fanelli, A stochastic reaction scheme for drug/metabolite interaction, Journal of Theoretical Biology (2009), doi:10.1016/j.jtbi.2009.03.031

This is a PDF file of an unedited manuscript that has been accepted for publication. As a service to our customers we are providing this early version of the manuscript. The manuscript will undergo copyediting, typesetting, and review of the resulting galley proof before it is published in its final citable form. Please note that during the production process errors may be discovered which could affect the content, and all legal disclaimers that apply to the journal pertain. 


\title{
A stochastic reaction scheme for drug/metabolite interaction
}

\author{
F. Di Patti ${ }^{\mathrm{a}, \mathrm{c}, \mathrm{b}, *}$, D. Fanelli ${ }^{\mathrm{b}, \mathrm{c}}$ \\ ${ }^{a}$ CSDC-Centro Interdipartimentale per lo Studio di Dinamiche Complesse, Università degli \\ Studi di Firenze, Italy \\ ${ }^{b}$ Dipartimento di Energetica, Università degli Studi di Firenze,Italy \\ ${ }^{c}$ INFN-Sezione di Firenze
}

\begin{abstract}
We present a simplified stochastic model to investigate the mechanisms of action of tramadol, a centrally acting analgesic, used for treating pain. The model accounts for the process of metabolization through the cytochrome CYP2D6 and the interactions between molecules and target receptors. The proposed formulation is stochastic in nature and allows to speculate on the role of finite-size fluctuations. Analytically, the master equation, governing the process under scrutiny, is derived and studied in the mean-field limit. The analysis of the associated asymptotic behavior proves interesting for its potential medical implications. The analysis of fluctuations is carried on via the van Kampen expansion. Numerical simulations are also performed to confirm the adequacy of our theoretical prediction.
\end{abstract}

Key words: Stochastic noise, Finite-size effects, Non-linear dynamics

\section{Introduction}

In recent years pharmacological research has moved towards the so called personalized medicine. This discipline combines data from genotype, gene expression and other clinical information, so to plan for an administered medical treatment which is targeted to a specific individual. In this respect, one hopes to maximize effectiveness of the therapy, while minimizing adverse, toxic reactions.

One of the crucial aspects to be accounted for when aiming at a personalized pharmacological treatment is the fact that drugs can undergo a process of metabolic transformation which either inactivate the substances, or, alternatively, gives rise to metabolites, which are occasionally more effective than the parent compound. The enzymes involved in metabolism are present in many tissues, but generally more concentrated in the liver (Katzung, 2003). Among

\footnotetext{
* Corresponding author

Email addresses: f.dipatti@gmail.com (F. Di Patti)
} 
drug metabolizing hepatic enzymes, cytochrome P450 (CYP) superfamily enclose the most prominent ones. It in fact accounts for the $75 \%$ of the total metabolism (Guengerich, 2008). This family is characterized by a high genetic variability: The Human Genome Project has identified 57 human genes coding for the various cytochrome $\mathrm{P} 450$ enzymes $^{1}$. These variations may substantially affect the individual response to the therapy, as commonly experienced in the medical practice. Detecting genetic variations in drug-metabolizing enzymes becomes e.g. essential for identifying individuals who can present adverse drug reactions to standard doses of certain medications. Individuals carrying cytochrome poor metabolizer variants exhibit different pharmacokinetics properties as compared to control individuals. As a result, non-conventional doses of medications may be eventually required to sustain the involved cytochrome activity for biotransformation. Conversely, medications that are not processed via cytochrome biotransformation, can be preferentially selected for those patients with potentially impaired cytochrome metabolic capacity.

A typical example of a drug which is metabolized by hepatic cytochromes is tramadol, a synthetic opioid widely used in the treatment of acute and chronic pain (Grond and Sablotzki, 2004). Tramadol undergoes hepatic metabolism via different isozymes of the cytochrome $\mathrm{P} 450$, being $\mathrm{O}$ - and $\mathrm{N}$-demethylated to five metabolites. Of these, the one catalyzed by cytochrome CYP2D6, called M1 (O-Desmethyltramadol), is the most significant since it displays a 200 fold increase in affinity, versus the $\mu$-opioid receptors, as compared to the tramadol. The process of metabolization results in different ways of action of tramadol on the peripheral and central nervous system, since the various metabolites bind to different specific receptors. The wide variability in the pharmacokinetic properties of tramadol, however, can only be partly ascribed to the CYP polymorphism. It is in fact clear that also fluctuations in the concentrations of tramadol, and its active metabolites, may impact the therapeutic response and toxicity (Grond and Sablotzki, 2004).

Such a complex picture points to the need for a comphrensive interpretative framework, where modeling efforts should develop well beyond the boundaries of standard pharmacology. Indeed, one would aim at disposing of detailed mathematical models which incorporate sufficiently accurate microscopic descriptions of the system at hand. This achievement would in turn enables researchers to resolve, within a self-consistent picture, the intricate cascade of reaction which mediates pain perception, so assisting medical doctors in designing the optimal, personalized, pharmacological treatment.

In this paper we take a first step in this direction by proposing and then analyzing a simplistic model which describes the process of metabolization of the tramadol. The interaction between the drug molecules and the target receptors is also accounted for. Although we here make specific reference to the case of tramadol, the model discussed is rather general and can hence be invoked within other contexts where metabolization and ligand-receptors interactions do occur.

\footnotetext{
${ }^{1}$ See http://drnelson.utmem.edu/human.P450.table.html
} 
The outline of the paper is the following. In the next section we introduce the stochastic model in term of associated chemical equations. The underlying master equation is also specified. In section 3, we recover the mean-field system which formally applies to the limit of infinite microscopic constituents. The fixed points of the mean-field model are studied, as well as their associated stability. As we shall pinpoint in the following, depending on the chemical parameters the drug act with a different degree of effectiveness, that we here quantify. Also, the transient dynamics present intriguing features, that we bring into evidence. Section 4 is devoted to investigating the role of fluctuations which are analytically studied via the van Kampen's expansion. Numerical simulations are performed to corroborate our findings. In the final section we sum up and draw our conclusions.

\section{Description of the model}

The bloodstream in the vicinity of the target receptor is assumed to be populated by two species of molecules, the parent drug tramadol and the main metabolite M1. Notice that, in general, metabolization and binding events occur in different parts of the body. Diffusion between sequentially ordered compartments should be in principle considered. In the present formulation space is however not explicitly incorporated and the reactions happen, according to their associated probability, within a unique bulk where molecules are uniformly stirred. As anticipated, the solely biological processes here addressed are hence the metabolization and the reversible chemical reactions between the molecules and the free target receptors. As a side comment, we also emphasize that competition with other molecular entities dispersed in the medium could be possibly included in the picture. This important aspects are discussed in (Di Patti and Fanelli, 2008, 2009).

The process of biotransformation through the cytochromes gives rise to the metabolites. Here we hypothesize that the cytochromes are present in great quantity in the body, so that metabolization does not depend on their associated concentration, and proceeds as a spontaneous transformation at constant rate. Denoting with $T$ the molecule of tramadol, and assuming $M$ to label the metabolite of type M1, the process of metabolization is reconducted to the following chemical reaction

$$
T \stackrel{\alpha}{\longrightarrow} M
$$

where $\alpha$ is the reaction constant. This is the parameter which quantifies the ability of the body to metabolize the drug and can be hence supposed to be intimately connected to the genetic polymorphisms of the cytochrome CYP2D6.

Tramadols sailing in the bloodstream can eventually encounter a free target receptor, hereafter labeled $R_{F}$. Following a successful binding event the receptor $R_{F}$ changes into an occupied element $R_{T}$. In formulae:

$$
T+R_{F} \stackrel{\beta_{1}}{\longrightarrow} R_{T}
$$




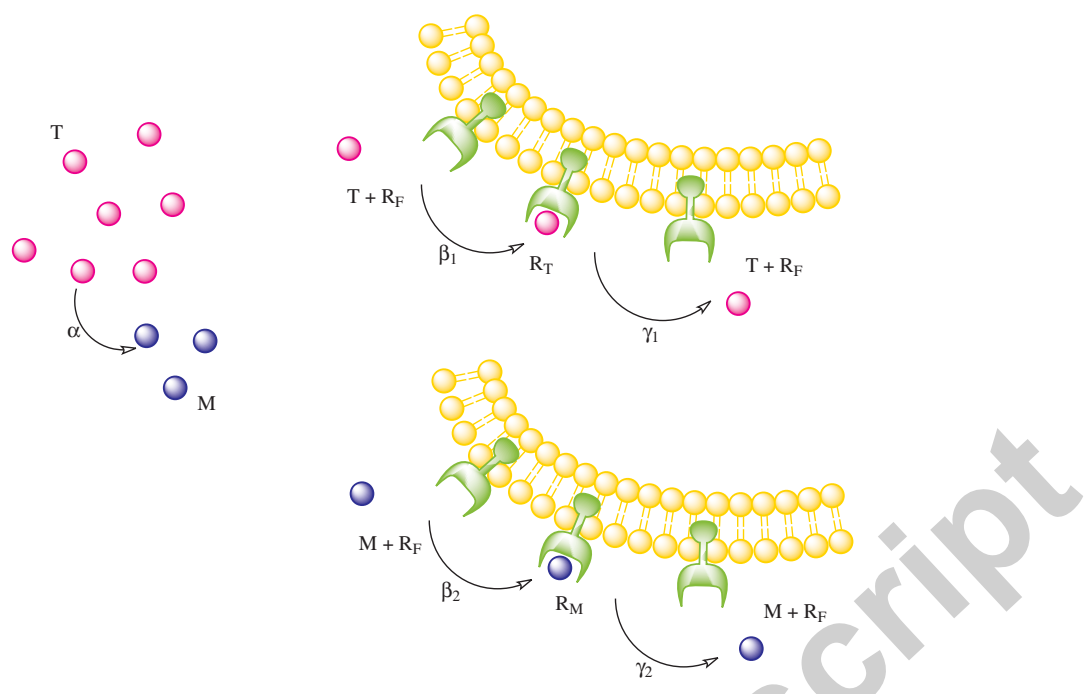

Figure 1: Chemical equations and schematic representation of the model. A tramadol molecule $(T)$ turns into metabolite $(\mathrm{M})$ with rate $\alpha$, and it can bind to a free receptor $R_{F}$ with rate $\beta_{1}$. The spontaneous detachment of the compound $R_{T}$ occurs with rate $\gamma_{1}$. Molecules of type $M$ bind and unbind to $R_{F}$ with rate $\beta_{2}$ and $\gamma_{2}$.

We further assume that during the binding the chemical properties of $T$ remain unaltered. It may hence occasionally occur that $R_{T}$ undergoes the inverse transformation by realising a, still active, $T$ molecule. This assumption translates into

$$
R_{T} \stackrel{\gamma_{1}}{\longrightarrow} T+R_{F}
$$

The two parameters $\beta_{1}$ and $\gamma_{1}$ are the constant reaction rates.

To complete the model, we have to include the interaction between metabolites and receptors. The medical literature reports on the specificity of tramadol and metabolites to the different classes of receptors involved in pain mechanism, and their role in achieving analgesia. Here, we set down to consider a simplified scenario, where the parent drug and metabolite bind to the same type of receptors. More specifically, we shall assume, in analogy with the above, the following reactions' scheme for $M$ :

$$
\begin{aligned}
& M+R_{F} \stackrel{\beta_{2}}{\longrightarrow} R_{M} \\
& R_{M} \stackrel{\gamma_{2}}{\longrightarrow} M+R_{F}
\end{aligned}
$$

where now $R_{M}$ is the compound receptor-metabolite, and $\beta_{2}$ and $\gamma_{2}$ stand for the associated (forward and backward) rates.

The cartoon in figure 1 depicts the reactions network that we imagine to characterize our model.

How to quantify the sensation of pain within the proposed picture? Inspired to (Di Patti and Fanelli, 2008, 2009), we imagine that the more the bound recep- 
tors the less the experienced pain. In other words, the ideal condition where all available receptors were screened by a pool of injected drug ( $T$ or $M)$ molecules, would correspond to achieving complete analgesia. In future perspective the model could be complemented by accommodating for the signal transduction pathway, and so accurately representing the neural activity steps involved in the process.

Another comment is mandatory at this point. We have in fact deliberately decided to disregard the elimination of tramadol and metabolites from the body. Elimination is indeed crucial and leads to the final absorbing state where the concentration of $T$ and $M$ are both zero. However, and being at present interested with elucidating the local interaction of drugs and receptors, we hypothesised the elimination to proceed on a different (sensibly longer) time scale. Under this working assumption we do imagine to focus on a sequence of snapshots of the (relatively faster) interaction dynamics, where the global number of microscopic actors can be assumed as constant.

Moreover, we can certainly assume that the total number of receptors does not change with time (degradation is also happening with a different characteristic time). Denoting with $n_{i}$ the number of molecules belonging to the $i$-th species, for $i=T, M, R_{T}, R_{M}, R_{F}$, the following constrains are in conclusion put forward:

$$
\begin{aligned}
n_{T}+n_{M}+n_{R_{T}}+n_{R_{M}} & =N_{1} \\
n_{R_{F}}+n_{R_{T}}+n_{R_{M}} & =N_{2}
\end{aligned}
$$

where $N_{1}$ represents the total number of molecules, while $N_{2}$ refers to the total number of receptors. We can use these relations to express $n_{R_{M}}$ and $n_{R_{F}}$ in terms of the other independent variables, namely $n_{R_{M}}=N_{1}-n_{T}-n_{M}-n_{R_{T}}$ and $n_{R_{F}}=N_{2}-N_{1}+n_{T}+n_{M}$, so that the state of the system is given by the three dimensional vector $\underline{n}=\left(n_{T}, n_{M}, n_{R_{T}}\right)$.

Within this framework, we are able to write the transition probabilities for the system to go from initial state $\underline{n}$ to the final (allowed) state $\underline{n}$ '. Such a probability is labeled $T(\underline{n} \mid \underline{n})$. In our system only transitions from $\underline{n}$ to $\left(n_{T}-\right.$ $\left.1, n_{M}+1, n_{R_{T}}\right),\left(n_{T} \pm 1, n_{M}, n_{R_{T}} \mp 1\right)$ and $\left(n_{T}, n_{M} \pm 1, n_{R_{T}}\right)$ can take place. The corresponding nonzero $T(\underline{n} \prime \mid \underline{n})$ entries are

$$
\begin{aligned}
T\left(n_{T}-1, n_{M}+1, n_{R_{T}} \mid \underline{n}\right) & =\alpha n_{T} \\
T\left(n_{T}-1, n_{M}, n_{R_{T}}+1 \mid \underline{n}\right) & =\beta_{1} n_{T} \frac{\left(N_{2}-N_{1}+n_{T}+n_{M}\right)}{N} \\
T\left(n_{T}, n_{M}-1, n_{R_{T}} \mid \underline{n}\right) & =\beta_{2} n_{M} \frac{\left(N_{2}-N_{1}+n_{T}+n_{M}\right)}{N} \\
T\left(n_{T}+1, n_{M}, n_{R_{T}}-1 \mid \underline{n}\right) & =2 \gamma_{1} n_{R_{T}} \\
T\left(n_{T}, n_{M}+1, n_{R_{T}} \mid \underline{n}\right) & =2 \gamma_{2}\left(N_{1}-n_{T}-n_{M}-n_{R_{T}}\right)
\end{aligned}
$$

where $N=N_{1}+N_{2}$.

Transition probabilities allow us to write down a master equation which governs the time evolution of the probability $P(\underline{n}, t)$, namely the probability of 
having the system in state $\underline{n}$ at time $t$. The rate of change of $P(\underline{n}, t)$ is simply given by the sum of the transitions towards $\underline{n}$, minus the outward transitions propagating from that state. In mathematical notation:

$$
\begin{aligned}
\frac{\mathrm{d}}{\mathrm{d} t} P(\underline{n}, t)= & T\left(\underline{n} \mid n_{T}+1, n_{M}-1, n_{R_{T}}\right) P\left(n_{T}+1, n_{M}-1, n_{R_{T}}, t\right) \\
& +T\left(\underline{n} \mid n_{T}+1, n_{M}, n_{R_{T}}-1\right) P\left(n_{T}+1, n_{M}, n_{R_{T}}-1, t\right) \\
& +T\left(\underline{n} \mid n_{T}, n_{M}+1, n_{R_{T}}\right) P\left(n_{T}, n_{M}+1, n_{R_{T}}, t\right) \\
& +T\left(\underline{n} \mid n_{T}-1, n_{M}, n_{R_{T}}+1\right) P\left(n_{T}-1, n_{M}, n_{R_{T}}+1, t\right) \\
& +T\left(\underline{n} \mid n_{T}, n_{M}-1, n_{R_{T}}\right) P\left(n_{T}, n_{M}-1, n_{R_{T}}, t\right) \\
& -\left[T\left(n_{T}-1, n_{M}+1, n_{R_{T}} \mid \underline{n}\right)+T\left(n_{T}-1, n_{M}, n_{R_{T}}+1 \mid \underline{n}\right)\right. \\
& +T\left(n_{T}, n_{M}-1, n_{R_{T}} \mid \underline{n}\right)+T\left(n_{T}+1, n_{M}, n_{R_{T}}-1 \mid \underline{n}\right) \\
& \left.+T\left(n_{T}, n_{M}+1, n_{R_{T}} \mid \underline{n}\right)\right] P(\underline{n}, t)
\end{aligned}
$$

with null initial and boundary conditions.

We have by now formulated our discrete stochastic model and specified the transition probabilities between the admissible states. The (exact) master equation could be, in principle, solved to obtain a closed expression for the expected probability at time $t$. This task turns out impossible and one has to resort to approximate solution. In first place, as discussed in the next section, the mean-field limit (namely $N_{1} \rightarrow \infty, N_{2} \rightarrow \infty$ ) can be recovered. Then fluctuations around the mean-field dynamics could be considered via perturbative techniques. Notice that simulating the full stochastic model is instead possible by resorting to the celebrated Gillespie algorithm, as outlined below.

\section{The deterministic limit}

Multiplying both sides of equation (1) by $n_{T}$ and summing over all states, we obtain

$$
\begin{aligned}
\frac{\mathrm{d}}{\mathrm{d} t} \sum_{\underline{n}} n_{T} P(\underline{n}, t) & =\sum_{\underline{n}}\left[T\left(n_{T}+1, n_{M}, n_{R_{T}}-1 \mid \underline{n}\right)\right. \\
& \left.-T\left(n_{T}-1, n_{M}+1, n_{R_{T}} \mid \underline{n}\right)-T\left(n_{T}-1, n_{M}, n_{R_{T}}+1 \mid \underline{n}\right)\right] P(\underline{n}, t)
\end{aligned}
$$

where the summation variables have been shifted to simplify the expression. Substituting in for the transition rates and remembering that by definition $\sum_{\underline{n}} n_{T} P(\underline{n}, t)=\left\langle n_{T}\right\rangle$, we have

$$
\frac{\mathrm{d}}{\mathrm{d} t}\left\langle n_{T}\right\rangle=-\alpha\left\langle n_{T}\right\rangle-\frac{\beta_{1}}{N}\left\langle n_{T}\left(N_{2}-N_{1}+n_{T}+n_{M}\right)\right\rangle+2 \gamma_{1}\left\langle n_{R_{T}}\right\rangle .
$$



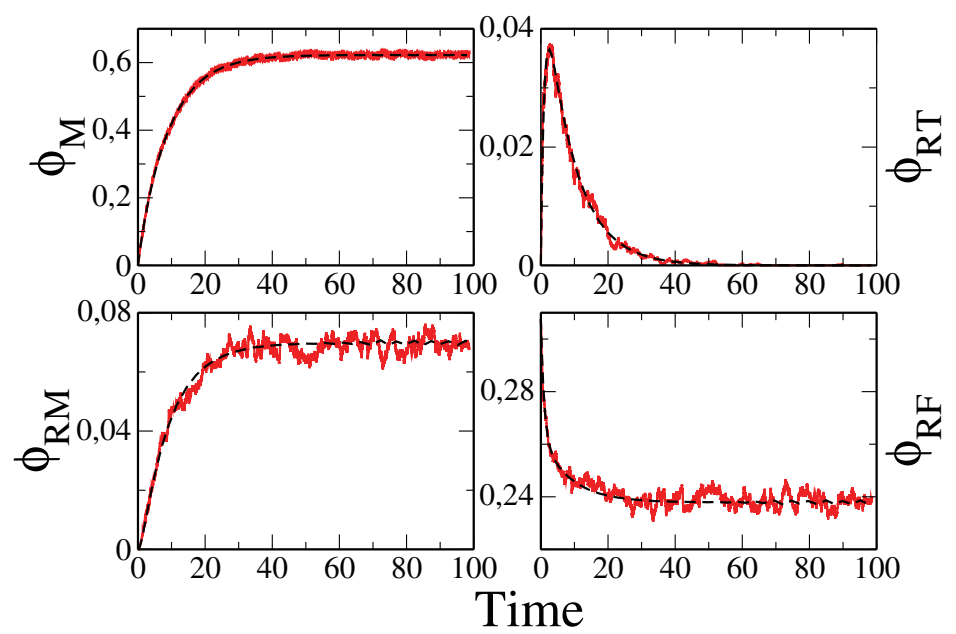

Figure 2: Metabolites and receptor densities as function of time. The solid (red) lines represent the stochastic simulation while the dashed (black) lines correspond to the numerical solution of the mean-field system. Parameters used are $\alpha=0.12, \beta_{1}=0.23, \beta_{2}=0.33, \gamma_{1}=0.4$, $\gamma_{2}=0.35, N_{1}=4500$ and $N_{2}=2000$.

Applying the same method to the two other variables, we obtain the following differential equations

$$
\begin{aligned}
\frac{\mathrm{d}}{\mathrm{d} t}\left\langle n_{M}\right\rangle= & \alpha\left\langle n_{T}\right\rangle-\frac{\beta_{2}}{N}\left\langle n_{M}\left(N_{2}-N_{1}+n_{T}+n_{M}\right)\right\rangle \\
& +2 \gamma_{2}\left\langle N_{1}-n_{T}-n_{M}-n_{R_{T}}\right\rangle \\
\frac{\mathrm{d}}{\mathrm{d} t}\left\langle n_{R_{T}}\right\rangle= & \frac{\beta_{1}}{N}\left\langle n_{T}\left(N_{2}-N_{1}+n_{T}+n_{M}\right)\right\rangle-2 \gamma_{1}\left\langle n_{R_{T}}\right\rangle .
\end{aligned}
$$

In the limit $N \rightarrow \infty$ we can replace $\left\langle n_{i} n_{j}\right\rangle=\left\langle n_{i}\right\rangle\left\langle n_{j}\right\rangle$ for every $i, j$ in equations (2)-(4). In this way $\left\langle n_{i}\right\rangle / N$ becomes the deterministic variable $\phi_{i}$, and we can write the mean-field system as:

$$
\begin{aligned}
& \frac{\mathrm{d}}{\mathrm{d} t} \phi_{T}=-\alpha \phi_{T}-\beta_{1} \phi_{T}\left(\sigma+\phi_{T}+\phi_{M}\right)+2 \gamma_{1} \phi_{R_{T}} \\
& \frac{\mathrm{d}}{\mathrm{d} t} \phi_{M}=\alpha \phi_{T}+2 \gamma_{2}\left(\varphi-\phi_{T}-\phi_{M}-\phi_{R_{T}}\right)-\beta_{2} \phi_{M}\left(\sigma+\phi_{T}+\phi_{M}\right) \\
& \frac{\mathrm{d}}{\mathrm{d} t} \phi_{R_{T}}=\beta_{1} \phi_{T}\left(\sigma+\phi_{T}+\phi_{M}\right)-2 \gamma_{1} \phi_{R_{T}}
\end{aligned}
$$

where $\sigma=\left(N_{2}-N_{1}\right) / N$ and $\varphi=N_{1} / N$.

Figure 2 shows the comparison between the stochastic behavior of the model and the mean-field one, as calculated by numerical integration of equations (5), dashed (black) line. The continuous (red) line is a typical stochastic simulation obtained through implementing the Gillespie's exact algorithm (Gillespie, 1976). For each species, the two profiles overlap well: The approximate mean-field 
theory and the stochastic simulation agree, a part from corrections due to the finiteness of the simulated medium. As $T$ is not continuously administered, its (number) concentration (as well that of $R_{T}$ ) decays to zero. Conversely, the densities of the other species settle down to a steady-state value. This latter value and its stability properties are calculated in the following, where simple speculations on the medical relevance of our findings are also going to be addressed.

\subsection{Analysis of the macroscopic equations}

To find the equilibrium point of the macroscopic equations, we set the time derivative to zero in system (5) and solve, obtaining the point $\phi^{*}=\left(0, \phi_{M}^{*}, 0\right)$ where

$$
\phi_{M}^{*}=\frac{-\left(2 \gamma_{2}+\beta_{2} \sigma\right)+\sqrt{\left(2 \gamma_{2}+\beta_{2} \sigma\right)^{2}+8 \gamma_{2} \varphi \beta_{2}}}{2 \beta_{2}} .
$$

The stability of this point can be deduced from the Jacobian matrix

$$
J\left(\phi^{*}\right)=\left(\begin{array}{ccc}
-\alpha-\beta_{1} \sigma-\beta_{1} \phi_{M}^{*} & 0 & 2 \gamma_{1} \\
\alpha-2 \gamma_{2}-\beta_{2} \phi_{M}^{*} & -2 \gamma_{2}-\beta_{2} \sigma-2 \beta_{2} \phi_{M}^{*} & -2 \gamma_{2} \\
\beta_{1} \sigma+\beta_{1} \phi_{M}^{*} & 0 & -2 \gamma_{1}
\end{array}\right)
$$

whose eigenvalues are

$$
\begin{aligned}
\lambda_{1} & =-\sqrt{\left(2 \gamma_{2}+\beta_{2} \sigma\right)^{2}+8 \gamma_{2} \varphi \beta_{2}} \\
\lambda_{2,3} & =\frac{-\left(2 \gamma_{1}+\alpha+\beta_{1} \sigma+\beta_{1} \phi_{M}^{*}\right) \pm \sqrt{\left(2 \gamma_{1}+\alpha+\beta_{1} \sigma+\beta_{1} \phi_{M}^{*}\right)^{2}-8 \gamma_{1} \alpha}}{2} .
\end{aligned}
$$

These values are clearly negative and real, proving the system has a globally stable equilibrium point.

As explained in section 2, we here assess the effectiveness of the pharmacological treatment by measuring the number of bound receptors. However, recalling that the initial dose of tramadol is completely metabolized at equilibrium, we shall be solely interested with the quantity $\phi_{R_{M}}^{*}$. To visualize the asymptotic stage of the evolution we refer to the plan $\left(\phi_{R_{F}}, \phi_{R_{M}}\right)$ and therein trace the bisectrix (dashed line in figure 3a). Above the diagonal, $\phi_{R M}^{*}>\phi_{R F}^{*}$, the drug works better and the patient experiences less pain. Such a condition realizes if

$$
N_{1}>\frac{\beta_{2}+4 \gamma_{2}}{2\left(\beta_{2}-2 \gamma_{2}\right)} N_{2}
$$

and $\beta_{2}>2 \gamma_{2}$. This means that the forward binding rate for the metabolite must be (at least) a factor two larger than the corresponding dissociation constant. Moreover, the initial dose of administered drug has to be chosen so that $N_{1}$ is larger than (at least) $N_{2} / 2$.

As it is shown in Figure 3a, the equilibrium point is confined on the line $N \phi_{R_{M}}^{*}+N \phi_{R_{F}}^{*}-N_{2}=0$, which also defines the subdomain of the plane which can be visited during the transient dynamics. Above that line in fact the positiveness of the variables is guaranteed. In figure $3 \mathrm{~b}$ we project the numerical 


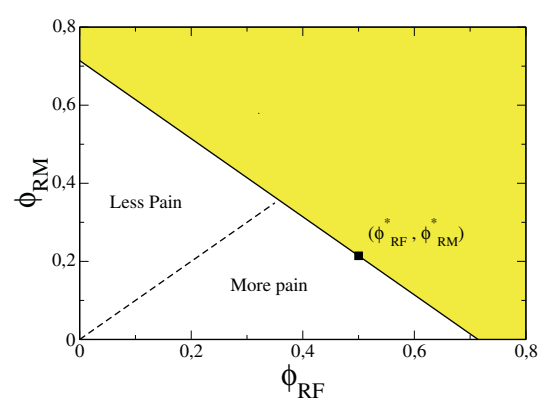

(a)

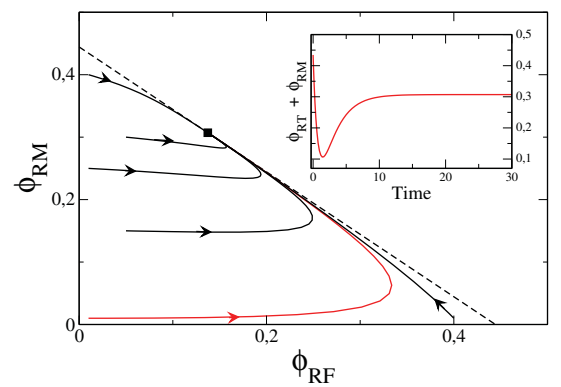

(b)

Figure 3: Panel (a) reports the projection of the equilibrium point into the plane $\phi_{R_{F}} \phi_{R_{M}}$. The two domains separated by the bisectrix are respectively labeled less/more pain, according to the prescriptions of the model (see main text). The colored (yellow) region denotes the portion of the plane where trajectories are not allowed. The solid black line corresponds to the condition $N \phi_{R_{M}}^{*}+N \phi_{R_{F}}^{*}-N_{2}=0$ (here $N_{1}=2000$ and $N_{2}=5000$ ). The equilibrium point (black square) belongs to this line. Panel (b) represents the projection of the trajectories on the plane $\phi_{R_{F}} \phi_{R_{M}}$ for different initial conditions. Parameters used for the numerical integration of the mean-field system are $\alpha=0.7, \beta_{1}=0.2, \beta_{2}=0.9, \gamma_{1}=0.8, \gamma_{2}=0.05$, $N_{1}=2500$ and $N_{2}=2000$. Inset: The time evolution of the total number of bound receptors, is reported. The curve is traced with reference to one specific initial condition, namely the red trajectory (online) of the main panel.

solutions of the system (5) in to the plane $\phi_{R_{F}}^{*} \phi_{R_{M}}^{*}$. The trajectories of the mean-field equations evolve towards the attractor. Starting from an arbitrary initial condition characterized by a generic value of $n_{R_{T}}$ and $n_{R_{M}}$, the system gets apparently trapped into a transient phase which displays an almost constant number of bound receptors, sensibly different from that eventually achieved at equilibrium. Indeed, as testified by figure $3 \mathrm{~b}$, the number of bound receptors initially shrinks and only after, due to the action of newly injected chemicals, starts growing to approach the fixed point. This setting could correspond to mimicking the condition where a patient is exposed to a treatment which closely follows a preceding drug administration.

Furthermore, the characteristic time of equilibration can be estimated as the (absolute value of the) inverse of the maximum eigenvalue (6)-(7). This is an interesting indicator as it quantifies the ability of the system to eventually attain the asymptotic condition where the largest number of receptors is occupied. The data in figure 4 show that the relaxation time decreases as the metabolization rate increases, thus suggesting that the administered drug acts more rapidly for extensive metabolizers.

\section{The van Kampen expansion}

Corrections to the mean-field dynamics can be calculated by resorting to the van Kampen's expansion (van Kampen, 1992). The main idea is to write the variables $n_{i}$ as a sum of two contributions, namely $n_{i}=N \phi_{i}(t)+\sqrt{N} \xi_{i}$, 


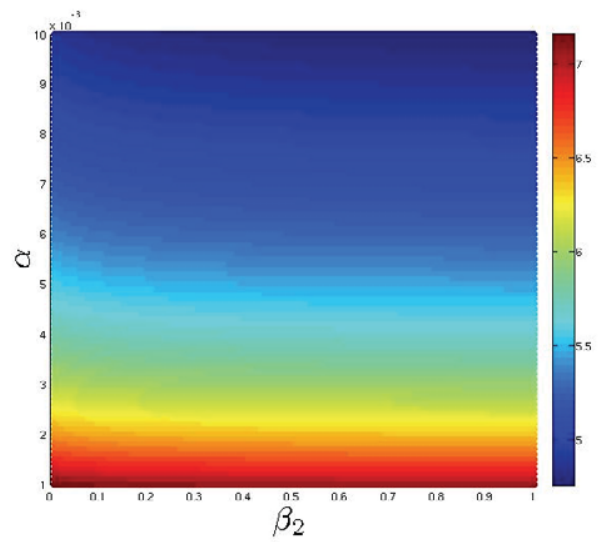

Figure 4: The color code (in logarithmic scale) refers to the slowest characteristic time of convergence to equilibrium as calculated from the linearized dynamics. We here scan the parameter plane $\left(\alpha, \beta_{2}\right)$. The other parameters are chose as $\beta_{1}=0.3, \gamma_{1}=0.5, \gamma_{2}=0.1$, $N_{1}=3000, N_{2}=2500$.

where $i=T, M, R_{T}$. Here $\phi_{i}$ stands for the deterministic component, while $\xi_{i}$ relates to the fluctuations. The scaling factor $1 / \sqrt{N}$ follows from the central limit theorem. A new probability distribution function $\Pi$ can be hence defined as $\Pi(\underline{\xi}, t)=P(\underline{n}, t)$ where $\underline{\xi}=\left(\xi_{T}, \xi_{M}, \xi_{R_{T}}\right)$. Moreover:

$$
\frac{d P}{d t}=\frac{\partial \Pi}{\partial t}-\sqrt{N} \sum_{i=T, M, R_{T}} \frac{\mathrm{d} \phi_{i}}{\mathrm{~d} t} \frac{\partial \Pi}{\partial \xi_{i}}
$$

To simplify the notation, it is practice to rewrite the master equation (1) in terms of step operators. These latter are defined through their action on an arbitrary function of $\underline{n}$ as:

$$
\begin{aligned}
& \mathbb{E}_{T}^{ \pm 1} f(\underline{n}, t)=f\left(n_{T} \pm 1, n_{M}, n_{R T}\right) \\
& \mathbb{E}_{M}^{ \pm 1} f(\underline{n}, t)=f\left(n_{T}, n_{M} \pm 1, n_{R T}\right) \\
& \mathbb{E}_{R_{T}}^{ \pm 1} f(\underline{n}, t)=f\left(n_{T}, n_{M}, n_{R T} \pm 1\right) .
\end{aligned}
$$

The master equation can be therefore cast in the form:

$$
\begin{aligned}
\frac{\mathrm{d}}{\mathrm{d} t} P(\underline{n}, t)= & \left(\mathbb{E}_{T}^{+1} \mathbb{E}_{M}^{-1}-1\right) \alpha n_{T} P(\underline{n}, t) \\
& +\left(\mathbb{E}_{T}^{+1} \mathbb{E}_{R_{T}}^{-1}-1\right) \frac{\beta_{1}}{N} n_{T}\left(N_{2}-N_{1}+n_{T}+n_{M}\right) P(\underline{n}, t) \\
& +\left(\mathbb{E}_{M}^{+1}-1\right) \frac{\beta_{2}}{N} n_{M}\left(N_{2}-N_{1}+n_{T}+n_{M}\right) P(\underline{n}, t) \\
& +\left(\mathbb{E}_{T}^{-1} \mathbb{E}_{R_{T}}^{+1}-1\right) 2 \gamma_{1} n_{R_{T}} P(\underline{n}, t) \\
& +\left(\mathbb{E}_{M}^{-1}-1\right) 2 \gamma_{2}\left(N_{1}-n_{T}-n_{M}-n_{R_{T}}\right) P(\underline{n}, t) .
\end{aligned}
$$


The operators $\mathbb{E}_{i}^{ \pm 1}$ change $n_{i}$ in $n_{i} \pm 1$ and so $\xi_{i}$ in $\xi_{i} \pm 1$. They hence admit the following representation in terms of differential operators:

$$
\mathbb{E}_{i}^{ \pm 1}=1 \pm N^{-1 / 2} \frac{\partial}{\partial \xi_{i}}+\frac{1}{2} N^{-1} \frac{\partial^{2}}{\partial \xi_{i}^{2}} \pm \cdots
$$

Substituting relation (9) into (8) and collecting contributions of order $\sqrt{N}$, one recovers the mean-field system of coupled differential equations (5). Working out the next-to-leading order, namely $N$, one eventually obtains a Fokker Planck equation (FPE) which characterizes the fluctuations around the asymptotic mean-field solution. The FPE reads:

$$
\frac{\partial \Pi}{\partial \tau}=-\sum_{i} \frac{\partial}{\partial \xi_{i}}\left(A_{i}(\underline{\xi}) \Pi\right)+\frac{1}{2} \sum_{i j} B_{i j} \frac{\partial^{2} \Pi}{\partial \xi_{i} \partial \xi_{j}}
$$

where

$$
A\left({ }_{i} \underline{\xi}\right)=\sum_{j} M_{i j} \xi_{j}
$$

The entries of matrix $M$ and $B$ are given in the appendix as a function of the chemical parameters of the model. Equation (10) can be solved explicitly: The obtained probability distribution $\Pi(\underline{\xi}, t)$ is a Gaussian and it is hence completely specified by its first and second moments. In the next section we shall calculate the associated moments explicitly and test the adequacy of the predictions versus direct simulations.

\subsection{Analysis of the fluctuations}

To characterize the moments of the distribution we proceed as follows. We multiply both sides of the FPE (10) by $\xi_{i}\left(\right.$ resp. $\left.\xi_{i} \xi_{j}\right)$ and integrate over all $\underline{\xi}$. One then recovers the equations for the mean value of the fluctuations $\left\langle\xi_{i}\right\rangle$, as well as for the associated correlations, $\left\langle\xi_{i} \xi_{j}\right\rangle$.

The evolution of the first moments is found to be governed by the following equations:

$$
\begin{aligned}
\frac{\mathrm{d}}{\mathrm{d} t}\left\langle\xi_{T}\right\rangle & =m_{11}\left\langle\xi_{T}\right\rangle+m_{12}\left\langle\xi_{M}\right\rangle+m_{13}\left\langle\xi_{R_{T}}\right\rangle \\
\frac{\mathrm{d}}{\mathrm{d} t}\left\langle\xi_{M}\right\rangle & =m_{21}\left\langle\xi_{T}\right\rangle+m_{22}\left\langle\xi_{M}\right\rangle+m_{23}\left\langle\xi_{R_{T}}\right\rangle \\
\frac{\mathrm{d}}{\mathrm{d} t}\left\langle\xi_{R_{T}}\right\rangle & =m_{31}\left\langle\xi_{T}\right\rangle+m_{32}\left\langle\xi_{M}\right\rangle+m_{33}\left\langle\xi_{R_{T}}\right\rangle
\end{aligned}
$$


while the the second moments obey to

$$
\begin{aligned}
\frac{\mathrm{d}}{\mathrm{d} t}\left\langle\xi_{T}^{2}\right\rangle= & 2 m_{11}\left\langle\xi_{T}^{2}\right\rangle+m_{12}\left\langle\xi_{T} \xi_{M}\right\rangle+2 m_{13}\left\langle\xi_{T} \xi_{R_{T}}\right\rangle+b_{11} \\
\frac{\mathrm{d}}{\mathrm{d} t}\left\langle\xi_{T} \xi_{M}\right\rangle= & m_{21}\left\langle\xi_{T}^{2}\right\rangle+\left(m_{11}+m_{22}\right)\left\langle\xi_{T} \xi_{M}\right\rangle+m_{23}\left\langle\xi_{T} \xi_{R_{T}}\right\rangle \\
& +m_{12}\left\langle\xi_{M}^{2}\right\rangle+m_{13}\left\langle\xi_{M} \xi_{R T}\right\rangle+b_{12} \\
\frac{\mathrm{d}}{\mathrm{d} t}\left\langle\xi_{T} \xi_{R T}\right\rangle= & m_{31}\left\langle\xi_{T}^{2}\right\rangle+m_{32}\left\langle\xi_{T} \xi_{M}\right\rangle+\left(m_{11}+m_{33}\right)\left\langle\xi_{T} \xi_{R_{T}}\right\rangle \\
& +m_{12}\left\langle\xi_{M} \xi_{R T}\right\rangle+m_{13}\left\langle\xi_{R T}^{2}\right\rangle+b_{13} \\
\frac{\mathrm{d}}{\mathrm{d} t}\left\langle\xi_{M}^{2}\right\rangle= & 2 m_{21}\left\langle\xi_{T} \xi_{M}\right\rangle+2 m_{22}\left\langle\xi_{M}^{2}\right\rangle+2 m_{23}\left\langle\xi_{M} \xi_{R_{T}}\right\rangle+b_{22} \\
\frac{\mathrm{d}}{\mathrm{d} t}\left\langle\xi_{M} \xi_{R T}\right\rangle= & m_{31}\left\langle\xi_{T} \xi_{M}\right\rangle+m_{21}\left\langle\xi_{T} \xi_{R T}\right\rangle+m_{32}\left\langle\xi_{M}^{2}\right\rangle+\left(m_{22}+m_{33}\right)\left\langle\xi_{M} \xi_{R_{T}}\right\rangle \\
& +m_{23}\left\langle\xi_{R T}^{2}\right\rangle \\
\frac{\mathrm{d}}{\mathrm{d} t}\left\langle\xi_{R T}^{2}\right\rangle= & 2 m_{31}\left\langle\xi_{T} \xi_{R_{T}}\right\rangle+2 m_{32}\left\langle\xi_{M} \xi_{R T}\right\rangle+2 m_{33}\left\langle\xi_{R T}^{2}\right\rangle+b_{33}
\end{aligned}
$$

where elements $m_{i j}$ and $b_{i j}$ are listed in the appendix.

The above system cannot be solved analytically (indeed we cannot even cast the mean-field solution in a closed analytic form). However, being interested in the fluctuations around the equilibrium point, once the initial transient has damped out, one sets to zero the time derivatives in system (11) and evaluates the coefficients $m_{i j}$ at the equilibrium point $\underline{\phi}^{*}$. It turns out that $\left\langle\xi_{T}\right\rangle^{s t}=$ $\left\langle\xi_{M}\right\rangle^{s t}=\left\langle\xi_{R T}\right\rangle^{s t}=0$. Proceeding in a similar fashion with system (12), one readily finds that all the second moments are zero but $\left\langle\xi_{M}^{2}\right\rangle^{s t}$ which instead reads

$$
\left\langle\xi_{M}^{2}\right\rangle^{s t}=\frac{\beta_{2} \phi_{M}^{*}+\left(\beta_{2} \sigma-2 \gamma_{2}\right) \phi_{M}^{*}+2 \gamma_{2} \varphi}{2\left(2 \beta_{2} \phi_{M}^{*}+\beta_{2} \sigma+2 \gamma_{2}\right)}
$$

In this latter case the stationary probability distribution $\Pi(\underline{\xi})^{s t}$ is given by

$$
\Pi(\underline{\xi})^{s t}=\frac{1}{\sqrt{2 \pi}\left\langle\xi_{M}^{2}\right\rangle^{s t}} \exp \left[-\frac{\xi_{M}^{2}}{2\left\langle\xi_{M}^{2}\right\rangle^{s t}}\right]
$$

Figure 5 a shows the projection of the stationary probability distribution $\Pi^{s t}$ on the plane $\left(\phi_{R F}, \phi_{R M}\right)$. As it can be appreciated by visual inspection, the dispersion occurs along the direction given by $N \phi_{R_{M}}^{*}+N \phi_{R_{F}}^{*}-N_{2}=0$ which also contains the equilibrium point. In figure $5 \mathrm{~b}$ the stationary probability distribution $\Pi^{s t}$ is plotted as a function of $\xi_{M}$. The figure testifies on the predictive ability of equation (13) here depicted with a solid line, which is shown to interpolate correctly the numerical data (symbols).

Imagine now to partition the plane $\left(\phi_{R F}, \phi_{R M}\right)$ into two regions separated by the bisectrix. Moving above the diagonal, the number of screened receptors increases which in turn implies reducing the pain, within our simplified scenario. Fluctuations can facilitate the road towards analgesia, as outlined in figure $5 \mathrm{a}$. 


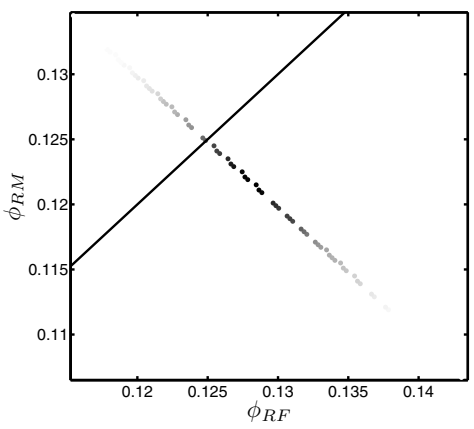

(a)

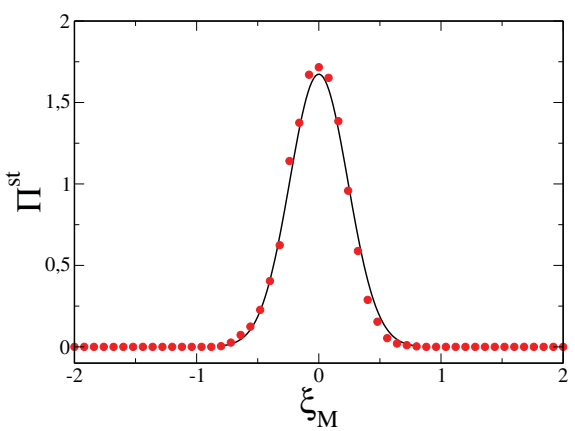

(b)

Figure 5: Panel (a) shows the projection of the stationary probability distribution $\Pi^{s t}$ on the original plane $\phi_{R F} \phi_{R M}$. The solid black line represents the bisectrix of the plane. Panel (b) reports the stationary probability distribution as function of $\xi_{M}$. The solid line represents the theoretical prediction based on equation (13). The symbols refer to the Gillespie like numerical simulation. Here, the parameters are $\alpha=0.5, \beta_{1}=0.2, \beta_{2}=0.3, \gamma_{1}=0.5$, $\gamma_{2}=0.1, N_{1}=3000$, and $N_{2}=1000$.

The stationary probability distribution can be be hypothetically employed to quantify the probability of entering the region in $\left(\phi_{R F}, \phi_{R M}\right)$ where the drug effect is supposedly more pronounced. This latter probability corresponds to the area of the distribution above the bisectrix and is quantified in $p=0.196$ for the chosen parameters' setting. Interestingly, although the mean-field solution predicts a stationary condition characterized by a pronounced sensation of pain $\left(\phi_{R F}^{*}=0.129\right.$ and $\left.\phi_{R M}^{*}=0.121\right)$, there is a nonzero probability that, due to fluctuations, the system enters a region where pain is partially hindered. Obviously, this speculation applies as long as $N$ is finite (though large). This is for instance the case where a local stimulus is applies which interests a finite patch of neurons (see for instance the whisker stimulation experiment (Rojas et al., 2006)). In the general case where the number of involved receptors is exceedingly large, the mean-field dynamics takes over and the aforementioned distribution shrinks to a delta.

\section{Conclusions}

In this paper we present a stochastic model which describes the mechanism of action of tramadol, a synthetic opioid widely used in the management of chronic and acute pain. The proposed formulation consists of two populations of molecules, the parent drug and metabolites: Parent drug produces metabolites through metabolization, and both kind of molecules interact with the same target receptors so to induce analgesia. Within our simplified context, we choose to quantify the effectiveness of the pharmacological treatment by measuring the number of receptors instantaneously bound to the active molecules. 
The system is analyzed, focusing first on the mean-field dynamics $(N \rightarrow \infty)$ which is governed by a set of coupled ordinary differential equations for the species amount. The fixed points are studied together with the associated stability properties. The chemical parameters are found to control the asymptotic regime determining the effectiveness of the administered therapy. More interestingly, in the transient dynamics a lethargic phase is registered, where the number of bound receptors appear to have stabilized to a quota sensitive to initial condition, before reaching their equilibrium solution.

Moreover, fluctuations have been also analyzed via the van Kampen technique. It is here speculated that, in particular cases, they might influence the degree of experienced pain, which could hence change over time.

\section{Appendix}

In this Appendix we list the entries of the matrices $M$ and $B$ as calculated via the van Kampen expansion. The elements of the matrix $M$, hereafter $m_{i j}$, are

$$
\begin{aligned}
& m_{11}=-\left[\alpha+2 \beta_{1} \phi_{T}+\beta_{1} \sigma+\beta_{1} \phi_{M}\right] \\
& m_{12}=-\beta_{1} \phi_{T} \\
& m_{13}=2 \gamma_{1} \\
& m_{21}=\alpha-\beta_{2} \phi_{M}-2 \gamma_{2} \\
& m_{22}=-\left(2 \beta_{2} \phi_{M}+\beta_{2} \sigma+\beta_{2} \phi_{T}+2 \gamma_{2}\right) \\
& m_{23}=-2 \gamma_{2} \\
& m_{31}=2 \beta_{1} \phi_{T}+\beta_{1} \sigma+\beta_{1} \phi_{M} \\
& m_{32}=\beta_{2} \phi_{T} \\
& m_{33}=-2 \gamma_{1}
\end{aligned}
$$

The elements of matrix $\mathrm{B}$, hereafter $b_{i j}$, read

$$
\begin{aligned}
& b_{11}=\beta_{1} \sigma \phi_{T}+\beta_{1} \phi_{T}^{2}+\beta_{1} \phi_{T} \phi_{M}+\alpha \phi_{T}+2 \gamma_{1} \phi_{R_{T}} \\
& b_{12}=-\alpha \phi_{T} \\
& b_{13}=-\left[\beta_{1} \sigma \phi_{T}+\beta_{1} \phi_{T}^{2}+\beta_{1} \phi_{T} \phi_{M}+2 \gamma_{1} \phi_{R_{T}}\right] \\
& b_{22}=\beta_{2} \phi_{M}^{2}+\beta_{2} \sigma \phi_{M}+\beta_{2} \phi_{M} \phi_{T}+2 \gamma_{2} \varphi-2 \gamma_{2} \phi_{T}-2 \gamma_{2} \phi_{M}-2 \gamma_{2} \phi_{R_{T}}+\alpha \phi_{T} \\
& b_{23}=0 \\
& b_{33}=\beta_{1} \phi_{T} \sigma+\beta_{1} \phi_{T}^{2}+\beta_{1} \phi_{T} \phi_{M}+2 \gamma_{1} \phi_{R_{T}}
\end{aligned}
$$

\section{References}

Di Patti, F., Fanelli, D., 2008. Can a microscopic stochastic model explain the emergence of pain cycles in patients?, doi: 10.1088/1742$5468 / 2009 / 01 / \mathrm{P} 01004$. 
Di Patti, F., Fanelli, D., 2009. On the molecular mechanisms driving pain perception and emergent collective behaviors, submitted to EJPB.

Gillespie, D., 1976. A general method for numerically simulating the stochastic time evolution of coupled chemical reactions. Journal of Computational Physics 22, 403-434.

Grond, S., Sablotzki, A., 2004. Clinical pharmacology of tramadol. Clinical Pharmacokinetics 43 (13), 879-923.

Guengerich, F., 2008. Cytochrome P450 and chemical toxicology. Chem Res Toxicol 21, 70-83.

Katzung, B., 2003. Basic \& Clinical Pharmacology. McGraw-Hill Medical.

Rojas, M., Navas, J., Rector, D., 2006. Evoked response potential markers for anesthetic and behavioral states. Am J Physiol Regul Integr Comp Physiol 291, R189-R196.

van Kampen, N., 1992. Stochastic preocesses in Physics and Chemistry. North Holland, Amsterdam. 